\title{
Téoros
}

Revue de recherche en tourisme

\section{Clientèle américaine} On repart à zéro

\section{Claude Péloquin}

Volume 23, numéro 1, printemps 2004

URI : https://id.erudit.org/iderudit/1071372ar

DOI : https://doi.org/10.7202/1071372ar

Aller au sommaire du numéro

Éditeur(s)

Université du Québec à Montréal

ISSN

0712-8657 (imprimé)

1923-2705 (numérique)

Découvrir la revue

Citer cette note

Péloquin, C. (2004). Clientèle américaine : on repart à zéro. Téoros, 23(1), 79-81.

https://doi.org/10.7202/1071372ar

Ce document est protégé par la loi sur le droit d'auteur. L’utilisation des services d’Érudit (y compris la reproduction) est assujettie à sa politique d'utilisation que vous pouvez consulter en ligne.

https://apropos.erudit.org/fr/usagers/politique-dutilisation/
Cet article est diffusé et préservé par Érudit.

Érudit est un consortium interuniversitaire sans but lucratif composé de l’Université de Montréal, l’Université Laval et l'Université du Québec à Montréal. Il a pour mission la promotion et la valorisation de la recherche. https://www.erudit.org/fr/ 


\section{Chaire de tourisme}

\section{Clientèle américaine \\ 0 n repart à zéro}

\section{Claude Péloquin}

$\mathbf{P}$ our le Canada, les États-Unis constituent le principal marché touristique international avec plus de 14 millions de visites d'une nuit ou plus chaque année (2 millions pour le Québec). Avec tout ce qui s'est passé au cours des 30 derniers mois, plusieurs s'interrogent quant à un retour à la normale au sein de l'industrie touristique, plus particulièrement en ce qui a trait au marché américain. Or, l'accumulation des événements bien connus a modifié la façon de penser des Américains et altéré fondamentalement l'attitude et le comportement du consommateur.

Yesawich, Pepperdine, Brown \& Russell ont étudié ce phénomène qu'ils ont appelé The New Normal. Selon cette firme, il faut repartir à zéro et définir de nouvelles bases de profil de la clientèle touristique américaine afin de mieux comprendre ce qui influencera son comportement de voyages à court terme. Ils identifient cinq tendances lourdes :

\section{Stressés}

Les Américains sont plus stressés que jamais et entrevoient l'avenir avec un certain scepticisme. Les indices de confiance des voyageurs ont atteint, en 2003, leur plus bas niveau depuis dix ans. Bien qu'il soit remonté de façon significative au premier trimestre 2004, ce niveau de confiance demeure très fragile et, surtout, volatile. Le rythme auquel se succèdent les mauvaises nouvelles les ont habitués à rester sur le qui-vive, contribuant à instaurer un climat de stress.

- $84 \%$ des Américains jugent qu'ils ont plus de soucis actuellement qu'il y a quelques années,

- $70 \%$ estiment que la vie est devenue trop compliquée,

- $60 \%$ ne disposent pas suffisamment de temps de vacances,

- $40 \%$ manquent de temps,

- $29 \%$ échangeraient une augmentation de salaire pour plus de vacances,

- $26 \%$ ont besoin de quelque chose pour se calmer,

- 15 millions pratiquent le yoga régulièrement.

Par ailleurs, selon l'économiste Pierre Fortin (2003) de l'UQAM, les Américains travaillent en moyenne $13 \%$ plus d'heures que les
Québécois et l'écart ne cesse de croître. La différence la plus importante réside dans la durée moyenne des vacances. La majorité de nos voisins du Sud s'offrent moins de deux semaines de vacances alors que, sur le Vieux Continent, les travailleurs bénéficient généralement de quatre semaines ou plus de vacances et ils sont encouragés à les prendre. Aux États-Unis, la période des vacances est « permise », mais souvent peu encouragée.

La combinaison de ces éléments incite les Américains à fuir leur environnement immédiat, ne serait-ce que pour de courtes périodes, pour refaire le plein et se distraire.

\section{Centrés sur la famille}

Les événements du 11 septembre 2001 ont ravivé les valeurs familiales des Américains. Le temps passé avec les être chers est devenu une denrée précieuse :

- $80 \%$ des parents souhaitent passer davantage de temps en famille,

- $40 \%$ des touristes font au moins un voyage avec leurs enfants,

- $60 \%$ des parents sont favorables à l'idée de faire un voyage avec leurs enfants durant la période scolaire, ce qui signifie que cette période n'est pas un frein pour la prise de vacances en famille,

- $21 \%$ des grands-parents font au moins un voyage avec leurs petits-enfants sans la présence des parents.

Ce rapprochement familial place les produits touristiques à caractère familial à l'avant-scène. Cela ouvre aussi la porte aux vacances multigénérationnelles de plus en plus populaires, permettant aux proches de passer du temps dans un contexte agréable. Certains croisiéristes et parcs à thème misent beaucoup sur ce segment de marché. Mentionnons toutefois que, lors du choix des vacances familiales, le rayon de déplacement et le budget alloué peuvent s'avérer limités.

\section{Exigeants}

Le consommateur n'a jamais été aussi bien informé. Internet joue évidemment un immense rôle dans la démocratisation de l'information. Résultat : nous avons affaire à une clientèle des plus exigeantes, tant en ce qui concerne la qualité que le prix :

- $80 \%$ tentent toujours de négocier le meilleur tarif disponible lors d'une réservation d'hôtel, 
- $80 \%$ sont disposés à modifier leurs plans de voyage s'ils trouvent de l'hébergement de même qualité à meilleur prix,

- $70 \%$ des Américains se fient davantage à leur opinion qu'à celle d'un expert,

- $60 \%$ estiment que le personnel de service ne se soucie pas de leurs besoins,

- 60 \% jugent qu'il est très/extrêmement important d'obtenir le meilleur prix possible pour des vacances,

- $60 \%$ souhaitent acheter des produits et services qui reflètent leurs besoins individuels,

- $30 \%$ sont disposés à payer davantage pour obtenir ce qu'ils veulent.

La clientèle s'attend à être valorisée, à recevoir un traitement spécial et un service personnalisé. Même si l'aspect du prix est essentiel, c'est la qualité du produit qui fidélise la clientèle.

\section{Branchés}

Selon le Travel Industry Association of America (TIA), plus de 42 millions d'Américains ont acheté des produits touristiques en ligne en 2003, pour des dépenses d'environ 43 milliards de dollars. L'hébergement sera l'un des segments qui profitera le plus de la croissance du tourisme en ligne au cours des prochaines années. Mentionnons également que l'achat en ligne des forfaits touristiques gagne en popularité auprès des voyageurs.

- $64 \%$ des e-touristes américains sont plus intéressés par les forfaits personnalisés « dynamic packaging » que les forfaits traditionnels,

- $60 \%$ des Américains estiment que la principale conséquence de la venue d'Internet s'est traduite par un meilleur contrôle de l'information,

- $35 \%$ seulement des e-touristes se considèrent des clients « fidèles »,

- $24 \%$ de l'ensemble des principales composantes vendues en ligne en 2003 (l'avion, l'hébergement et la voiture de location) ont été achetés au cours d'une même transaction,

- L'«e-consumer » est âgé de 42 ans, dispose d'un revenu de ménage de 83400 dollars américains, effectue 3,7 voyages d'agrément par année et dépense 2900 \$US annuellement pour ses vacances.

Les paramètres pour saisir ce touriste «branché » sont différents de ce qu'ils étaient il y a cinq ans. Internet accentuera son influence sur des aspects tels que la transparence des prix et la personnalisation des produits touristiques ainsi que la flexibilité lors du processus de réservation des vacances.

\section{Patriotiques}

L'opinion publique américaine a rarement été aussi teintée de patriotisme ; les événements récents ont eu pour effet d'amplifier ce sentiment général.

- $61 \%$ des Américains jugent qu'ils devraient faire un effort particulier pour éviter de voyager vers des pays qui n'avaient pas supporté les États-Unis lors de la guerre en Irak,
- $61 \%$ affirment que vivre des expériences centrées sur l'histoire ou la culture américaine s'avère important lors de la planification des vacances d'été,

- $55 \%$ estiment que la plupart des Américains sont très / extrêmement patriotiques,

- $24 \%$ des Américains se considèrent extrêmement patriotiques, soit une augmentation de $5 \%$ depuis 1999, selon le Harwood Institute (Gallup, 2002).

La chaîne américaine CBS (2003) faisait mention de cet engouement dans ce qu'elle appelait : The Patriotic Travel Rush où tous les sites touristiques à saveur patriotique recevaient une hausse significative de visiteurs. Cette situation peut constituer une occasion de capitaliser sur des produits touristiques qui permettent aux Américains de renouer avec leur passé et par lesquels ils se reconnaissent ou se sentent interpellés (musées, sites historiques, etc.).

\section{Composer avec la nouvelle réalité}

Cette nouvelle réalité moins temporaire qu'on aurait pu le croire se traduit par des changements dans ce que recherche l'Américain comme expérience touristique. Par exemple, le cadre sécuritaire du lieu d'hébergement agit maintenant comme un attribut de grande importance lors du choix des vacances. Plus de $86 \%$ considèrent cet aspect très ou extrêmement important. Un tel état d'esprit peut être lié à une nouvelle tendance identifiée par certains spécialistes alarmistes vis-à-vis le portrait de la société américaine. Il s'agit du phénomène du bunkering qui représente un durcissement de la tendance du cocooning et qui s'appuie sur une vision défensive d'une société violente où la famille doit être protégée dans un environnement social « homogène » et sécuritaire (quartiers neufs dans des forteresses, systèmes de sécurité performants, etc.).

Les forfaits détentes et les centres de santé à l'intérieur de get away week-ends s'avèrent des produits porteurs dans ce contexte. On pourrait penser qu'une offensive marketing du type « you are worth it » ou « vous le méritez bien » s'avère une stratégie gagnante auprès de ce segment de marché.

Soulignons un autre élément intéressant, près de la moitié des Américains ne souhaitent pas nécessairement opter pour une destination qu'ils n'ont jamais visitée auparavant. Le caractère sécurisant et familier $\mathrm{du}$ « voisin du Nord » de même que sa proximité constituent ainsi des atouts. On peut également penser que des attractions orientées vers la famille et mettant en valeur l'apprentissage - musées, parcs à thèmes ou croisières, par exemple - pourraient tirer leur épingle du jeu.

Les destinations limitrophes « rubber tire » seront ainsi privilégiées pour les vacances, consolidant la possibilité pour les intervenants du Québec de cibler particulièrement le marché de la Nouvelle-Angleterre. La proportion des voyages effectués en voiture est passée de $77 \%$ à $81 \%$ de 2002 à 2003.

Le profil sociologique de l'Américain a sensiblement changé au cours des dernières années. Grâce à une meilleure compréhension 
des facteurs qui influencent son comportement, les entreprises touristiques seront davantage en mesure d'adapter leurs stratégies et de mettre à profit « le petit quelque chose » qui saura l'interpeller.

Claude Péloquin est analyste au Réseau de veille en tourisme de la Chaire de Tourisme de l'UQAM.

\section{Bibliographie}

CBS News, « Beat The Patriotic Travel Rush » [www.cbsnews.com], consulté le 27 juin 2003.

Expedia.com, « Expedia.com Survey Reveals Too Many Americans Put Quality Time on Standby » [www.expedia.com], consulté le 21 mai 2003.

Fortin, Pierre (2003), « Différences dans les heures annuelles travaillées par les habitants entre les États-Unis et le Canada », Observateur International de la productivité, $\mathrm{n}^{\circ} \quad 6$, printemps.

Fox News/Opinion Dynamics (2003), The Roper Center for Public Opinion Research, University of Connecticut, Sondage, avril.

Hospitality Trends, « Overworked Americans Find Simple Solutions To Maximizing Their Diminishing Vacation Time » [www.htrendscom], consulté le 25 septembre 2003.

Kissell, Joe, « Work Week and Vacation Variances Stress », Interesting Thing of the Day [itotd.com], consulté le 25 juillet 2003.

PhoCusWright, The PhoCusWright Consumer Travel Trends Survey Sixth Edition, The PhoCusWright Channel [www.phocuswright.com], consulté le février 2004.
Pocock, Kate, « Multi-Generational Vacations Include the Kids, the Grandkids, Friends, and Neighbours », Canoe Travel [travel.canoe.ca], consulté le 15 février 2004.

Sain, Gary C., « The New Normal », Yesawich, Pepperdine, Brown \& Russell [www.ypb.com], consulté le 10 mars 2003.

Sain, Gary C., « Travel Marketing in the $21^{\text {st }}$ Century - A Look Ahead », Yesawich, Pepperdine, Brown \& Russell [www.ypb.com], consulté le 2 octobre 2003.

Statistique Canada (2004), « Estimation du nombre de voyages d'une nuit et plus des non-résidents au Canada, selon la province d'entrée », Catalogue n ${ }^{\circ}$ 66-001.

The Colonial Williamsburg Foundation (2003), « Harris Interactive Nationwide Poll Finds Historic, Cultural Sites Important to 61 Percent of American Family Vacation Planners », 18 juin.

The Economist (2003), « A Holiday from History », 9 août, p. 45.

The Gallup Organization (2002), Harwood Institute Political Conduct Survey, Topline, 21 janvier.

Travel Industry Association of America, « TIA Shows Continued Growth in Online Travel Bookers; Nearly One-third Book all their Travel Online » [www.tia.org], consulté le 22 janvier 2004.

Yesawich, Peter (2003), « Superior Small Lodgings Conference in Fort Lauderdale », Extrait de conférence, 2003 Conference and Trade Show, 7-10 décembre.

YPBR \& Yankelovich Partners (2003), National Leisure Travel Monitor. Zernike, Kate (2003), « The Vanishing American Vacation », The New York Times, 29 août.

\section{Programmes de deuxième cycle en Gestion et planification du tourisme}

$\hookrightarrow$ Programme court de 5 cours offert à temps partiel, 2 ans

$\hookrightarrow$ Diplôme d'études supérieures spécialisées (D.E.S.S.)

$\rightarrow$ Maîtrise (M. Sc.) offerte à temps plein ou partiel, 2 ou 4 ans

Directrice : $\quad$ Danielle Pilette, Ph.D.

(514) 987-3000, poste 8544

Candidatures: pour l'automne 2004,

d'ici le $1^{\text {er }}$ mai;

pour l'hiver 2005,

d'ici le 15 octobre.

Conditions générales d'admission :

Diplôme de premier cycle et deux ans d'expérience pertinente

\section{UQĀM}

Prenez position 